\title{
Intact but not denatured ovine serum immunoglobulins positively modulate mucosal immune mediators in the growing rat challenged with Salmonella enteritidis
}

\author{
Prabhu Balan $^{1,2 *}$ and Paul J. Moughan ${ }^{1}$ \\ ${ }^{1}$ Riddet Institute, Massey University, Private Bag, 11222 Palmerston North, New Zealand \\ ${ }^{2}$ AgResearch Ltd, Ruakura Research Centre, Private Bag 3123, Hamilton, New Zealand \\ (Submitted 9 August 2012 - Final revision received 3 January 2013 - Accepted 5 January 2013 - First published online 22 February 2013 )
}

\section{Abstract}

Immunoglobulins are major glycoproteins that modulate the immune response of gut-associated lymphoid tissue. In the present study, we sought to determine whether orally administered ovine serum immunoglobulins modulate selected indices of mucosal immune function and immune mediators in the growing rat challenged with Salmonella enteritidis. Rats were fed a casein-based basal control diet (BD; unchallenged). Three groups of rats were challenged orally with $1 \times 10^{7}$ viable $S$. enteritidis on day 15 of the study and were fed the $\mathrm{BD}$, the BD containing freeze-dried ovine immunoglobulins (FDOI), or the BD containing autoclaved ovine immunoglobulins (AOI; negative control diet). The rats were randomly allocated to one of the four groups ( $n$ 15) and consumed their diet for $18 \mathrm{~d}$. In all of the intestinal segments, the challenged rats fed either the BD or AOI diet produced higher $(P<0.05)$ mucosal levels of interferon- $\gamma$, TNF $\alpha$, IgA and myeloperoxidase activity than the challenged rats fed the FDOI diet. In contrast, IL- 4 and IL-10 levels were higher in the challenged FDOI-fed rats compared with the other challenged groups. The challenged FDOI-fed rats had higher $(P<0 \cdot 05)$ mucosal anti-Salmonella IgA and IgG in all of the intestinal segments except the jejunum and ileum. Generally, the challenged rats receiving the FDOI diet had significantly $(P<0.05)$ higher mucosal mucin protein content compared with challenged rats receiving either the BD or AOI diet. In conclusion, an ovine immunoglobulin fraction positively modulated some selected indices of mucosal immune function and its mediators in growing rats challenged with $S$. enteritidis.

Key words: Ovine serum immunoglobulins: Cytokines: Gut mucosal immunity: Myeloperoxidase: Mucin

Immunoglobulins are a group of specialised bioactive proteins, present in blood plasma, serum and other tissue fluids, including the milk of all mammals ${ }^{(1)}$. Numerous studies have reported benefits from including animal plasma that contains immunoglobulins in the post-weaning diet of production animals, with improvements observed in feed intake, growth rate and intestinal organ growth ${ }^{(2,3)}$. The beneficial effect of animal plasma seems to be more pronounced in animals during a pathogen challenge $\mathrm{e}^{(4)}$. Lallès et $a{ }^{(5)}{ }^{(5)}$ reported that the beneficial effects of animal plasma were mediated mainly by its immunoglobulin fraction. The observed effects of the immunoglobulin fraction on growth in animals might be due to a greater availability of nutrients and energy for growth, consequent upon a lower degree of immune cell activation and an altered integrity and structure of the intestinal mucosa ${ }^{(6)}$.

Orally administered human immunoglobulins have been used as a prophylactic treatment for children born prematurely with necrotising enterocolitis ${ }^{(7)}$. Immunoglobulins have also been used therapeutically for medical intervention of Campylobacter jejuni ${ }^{(8)}$, Clostridium difficile ${ }^{(9)}$, Helicobacter pylori, rotavirus and cryptosporidial infection. Reports have shown that orally administered immunoglobulins retain activity after digestion in the gastrointestinal tract $^{(10)}$. We recently demonstrated in a rat model that oral administration of purified intact ovine (extracted from lamb's blood) serum immunoglobulins (and not the heat-denatured fraction: autoclaved ovine serum immunoglobulins) was effective in improving growth performance ${ }^{(11)}$ and led to a higher degree of immunological activity as evidenced by increased phagocytic activity, lymphocyte proliferation, cytokine production and intestinal immunoglobulin levels ${ }^{(12)}$. Feeding ovine serum immunoglobulins also resulted in an increase in gut mucin protein concentrations ${ }^{(13)}$ and supported the growth of beneficial lactic acid bacteria and prevented

Abbreviations: AOI, autoclaved ovine immunoglobulins; ASE, anti-Salmonella; BD, basal (control) diet; FDOI, freeze-dried ovine immunoglobulins; IFN $\gamma$, interferon- $\gamma$; MLN, mesenteric lymph node; MPO, myeloperoxidase; Th, T-helper.

*Corresponding author: Dr Prabhu Balan, fax +64 7838 5625, email Prabhu.Balan@agresearch.co.nz 
access to water at all times. During week 1, the rats were acclimatised to the cages and received the BD ad libitum. At the end of the acclimatisation period the rats were randomly allocated to the four experimental groups such that there were fifteen rats per group. The treatment and control groups were as follows: (1) non-challenged rats fed the basal control diet (BD); (2) rats challenged with $S$. enteritidis and fed the $\mathrm{BD}$; (3) rats challenged with $S$. enteritidis and fed the FDOI-containing diet; and (4) rats challenged with $S$. enteritidis and fed the AOI-containing diet (negative control). The rats received the respective diets ad libitum for an $18 \mathrm{~d}$ experimental period. On day 15 , the rats in groups 2,3 and 4 were orally administered with a single dose of $S$. enteritidis ATCC 13076 ( $1 \mathrm{ml}$ of saline solution containing 3\% sodium bicarbonate with $1 \times 10^{7}$ viable $S$. enteritidis). S. enteritidis (strain 1891, originally from the UK) was purchased from the New Zealand Reference Culture Collection - Environmental Science and Research. Before use, S. enteritidis was subcultured three times in LB (Luria-Bertani) broth (Difco). The exact viable count of $S$. enteritidis inoculum was determined by plating on brilliant green agar (Oxoid). Rats in group 1 (unchallenged, BD) received $1 \mathrm{ml}$ of saline solution without S. enteritidis.

\section{Chemical analysis}

Dietary DM, ash, crude protein, total fat and gross energy contents of the diet (Table 1) were determined according to methods described by AOAC ${ }^{(18)}$. The carbohydrate fraction was determined by difference using the formula $(100-$ (moisture + ether extract + ash + crude protein $)=$ carbohydrate $)$.

\section{Post-mortem procedure}

On day 18 of the experiment, the rats were anaesthetised by intraperitoneal injection $(0 \cdot 1 \mathrm{ml} / 100 \mathrm{~g}$ live weight $)$ of a mixture containing two parts acepromazine maleate BP ( $2 \mathrm{~g} / \mathrm{l})$, five parts ketamine $(100 \mathrm{~g} / \mathrm{l})$, one part xylazine $(100 \mathrm{~g} / \mathrm{l})$ and two parts sterile water. Rats were then killed by exsanguination. The entire small intestine from the pylorus to the ileo-caecal valve was removed, dissected free of mesenteric attachments, with the duodenum (upper 12\% from the pylorus end, not used in the present study) being isolated and with the rest being divided into two parts (equal in length), designated to approximate the jejunum (centre of the small intestine) and the ileum (anterior to the ileo-caecal valve). Deng et al. ${ }^{(19)}$ reported that the jejunum, ileum, caecum and colon are the primary sites of invasion in normal mice after oral infection with $S$. enteritidis. The entire jejunum and ileum were collected and washed intraluminally with PBS. The number of Peyer's patches was counted in the entire ileal segment and recorded. The same procedure was followed for the caecum and colon. The intestinal segments were cut longitudinally, and the mucosa scraped using glass slides. The mucus from each segment was suspended in $5 \mathrm{ml}$ PBS containing $1 \%$ protease inhibitor (Sigma) and centrifuged (HERAEUS, Fresco 17; Thermo Electron Corp.) at $5000 \boldsymbol{g}$ for $30 \mathrm{~min}$ at $4^{\circ} \mathrm{C}$ to remove debris and the clear supernatant fraction was stored at $-80^{\circ} \mathrm{C}$ for further analysis. The mesenteric lymph nodes (MLN) and pancreas were also removed and weighed. The remaining portion of the animal was weighed as the empty body weight. Relative weights of the pancreas were expressed relative to empty body weight.

Table 2. Intestinal mucosal interferon- $\gamma$ (INF $\gamma$ ), IL-4, IL-10 and TNF $\alpha$ concentrations in unchallenged rats fed the basal diet (BD) and rats challenged with Salmonella for $3 \mathrm{~d}$ and fed the BD, freeze-dried ovine immunoglobulin (FDOI) diet or autoclaved ovine immunoglobulin (AOI) diet (Mean values with their standard errors; $n 15$ )

\begin{tabular}{|c|c|c|c|c|c|c|}
\hline & \multirow{2}{*}{$\frac{\text { Unchallenged }}{\mathrm{BD}}$} & \multicolumn{3}{|c|}{ Challenged } & \multirow[b]{2}{*}{ SEM } & \multirow[b]{2}{*}{$P$} \\
\hline & & BD & FDOI & $\mathrm{AOI}$ & & \\
\hline \multicolumn{7}{|l|}{ Jejunal mucosa } \\
\hline INF $\gamma(\mu \mathrm{g} / \mathrm{l})$ & $0.14^{b}$ & $0.56^{\mathrm{a}}$ & $0.15^{b}$ & $0.48^{\mathrm{a}}$ & 0.010 & 0.036 \\
\hline TNF $\alpha(n g / l)$ & $184 \cdot 87^{b}$ & $769 \cdot 99^{\mathrm{a}}$ & $192 \cdot 83^{b}$ & $706 \cdot 37^{a}$ & 199.81 & 0.044 \\
\hline IL-4 (ng/l) & $118 \cdot 23^{b}$ & $89.54^{b}$ & $190 \cdot 25^{a}$ & $92 \cdot 35^{\mathrm{b}}$ & 28.45 & 0.035 \\
\hline IL-10 (ng/l) & $165 \cdot 08^{b}$ & $83.58^{c}$ & $330 \cdot 39^{a}$ & $80 \cdot 55^{\mathrm{C}}$ & $42 \cdot 79$ & 0.015 \\
\hline \multicolumn{7}{|l|}{ Ileal mucosa } \\
\hline INF $\gamma(\mu \mathrm{g} / \mathrm{I})$ & $0.19^{b}$ & $0.61^{a}$ & $0.17^{b}$ & $0.67^{\mathrm{a}}$ & 0.012 & 0.031 \\
\hline $\mathrm{TNF} \alpha(\mathrm{ng} / \mathrm{l})$ & $199 \cdot 72^{b}$ & $824.84^{\mathrm{a}}$ & $177 \cdot 68^{\mathrm{b}}$ & $791 \cdot 22^{\mathrm{a}}$ & 169.58 & 0.037 \\
\hline IL-4 (ng/l) & $123.59^{b}$ & $84.90^{b}$ & $202 \cdot 61^{a}$ & $87 \cdot 71^{\mathrm{b}}$ & $31 \cdot 25$ & 0.029 \\
\hline IL-10 (ng/l) & $310 \cdot 44^{b}$ & $88.94^{c}$ & $592 \cdot 75^{a}$ & $85.91^{\mathrm{C}}$ & $81 \cdot 29$ & 0.001 \\
\hline \multicolumn{7}{|l|}{ Caecal mucosa } \\
\hline INF $\gamma(\mu \mathrm{g} / \mathrm{I})$ & $0.16^{\mathrm{b}}$ & $0.71^{a}$ & $0 \cdot 16^{\mathrm{b}}$ & $0.70^{\mathrm{a}}$ & 0.013 & 0.035 \\
\hline $\mathrm{TNF} \alpha(\mathrm{ng} / \mathrm{l})$ & $187 \cdot 27^{b}$ & $784 \cdot 28^{a}$ & $161 \cdot 75^{\mathrm{b}}$ & $728 \cdot 46^{\mathrm{a}}$ & 129.54 & 0.041 \\
\hline IL-4 (ng/l) & $99.59^{b}$ & $79 \cdot 84^{\mathrm{b}}$ & $192 \cdot 48^{a}$ & $81 \cdot 38^{b}$ & 41.01 & 0.042 \\
\hline IL-10 (ng/l) & $310 \cdot 44^{b}$ & $78 \cdot 17^{\mathrm{C}}$ & $672 \cdot 75^{\mathrm{a}}$ & $95 \cdot 35^{\mathrm{C}}$ & $31 \cdot 81$ & 0.003 \\
\hline \multicolumn{7}{|l|}{ Colonic mucosa } \\
\hline INF $\gamma(\mu \mathrm{g} / \mathrm{I})$ & $0.10^{\mathrm{b}}$ & $0.55^{a}$ & $0.11^{\mathrm{b}}$ & $0.52^{\mathrm{a}}$ & 0.016 & 0.008 \\
\hline TNF $\alpha(n g / l)$ & $133.44^{\mathrm{b}}$ & $738.56^{a}$ & $101.41^{\mathrm{b}}$ & $774.94^{\mathrm{a}}$ & $118 \cdot 2$ & 0.017 \\
\hline IL-4 (ng/l) & $92 \cdot 38^{\mathrm{b}}$ & $74 \cdot 37^{b}$ & $179 \cdot 58^{a}$ & $71 \cdot 26^{b}$ & 39.78 & 0.040 \\
\hline $\mathrm{IL}-10$ (ng/l) & $228 \cdot 21^{\mathrm{b}}$ & $146 \cdot 71^{\mathrm{b}, \mathrm{c}}$ & $793.52^{\mathrm{a}}$ & $143.68^{b, c}$ & 98.58 & 0.006 \\
\hline
\end{tabular}

${ }^{a, b, c}$ Mean values within a row with unlike superscript letters were significantly different $(P<0.05)$. 
Analysis of mucosal cytokines, $\lg A, \lg G, \lg E$, and Salmonella-specific mucosal IgA and IgG

The concentrations of pro-inflammatory cytokines (INF $\gamma$ and TNF $\alpha$ ), anti-inflammatory cytokines (IL-4 and IL-10), rat IgA, $\operatorname{IgG}, \operatorname{IgE}$, and Salmonella-specific IgA and IgG in the intestinal mucosa were measured by sandwich ELISA as previously described $^{(14)}$.

\section{Determination of myeloperoxidase activity}

MPO activity was measured as an indicator of neutrophil infiltration (intestinal inflammation) in intestinal mucosal samples using a Myeloperoxidase Colorimetric Activity Assay Kit (Biovision) according to the manufacturer's instructions. One unit of MPO is defined as the amount of MPO that hydrolyses the substrate and generates taurine chloramine to consume $1 \cdot 0$ $\mu$ mol trinitrobenzene (TNB) per min at $25^{\circ} \mathrm{C}$.

\section{Quantitative analysis of mucin}

Mucin concentrations in the intestinal mucosa were measured by an enzyme-linked lectin assay using wheat germ agglutinin (Sigma) as the lectin ${ }^{(13,20)}$. The concentration of mucin in samples was calculated with reference to a porcine gastric mucin (Sigma) standard curve. Data were expressed as $\mu \mathrm{g}$ or mg mucin/l of supernatant fraction of mucosa suspended in a PBS solution.

\section{Statistical analysis}

For most variables there were fifteen rats per treatment, but in some cases a lower $(n$ 8) sample size was used. Data were checked for normality and results expressed as mean values with their standard errors. Data were analysed using a oneway ANOVA using the General Linear Models procedure of
SAS/PROC GLM (SAS version 9.1; SAS Institute Inc.) and means were compared using Tukey's test. Statistical significance was accepted at $P<0.05$.

\section{Results}

The effect of administration of $S$. enteritidis to the rats was acute. Most of the rats from the BD- and AOI-supplemented diet groups displayed symptoms of $S$. enteritidis infection such as nasal discharge and red crusts around the nostrils after $3 \mathrm{~d}$ of challenge. The study culminated after $3 \mathrm{~d}$ of challenge for ethical reasons. After challenge with $S$. enteritidis over $3 \mathrm{~d}$, there was a marked decrease in body weight and feed conversion efficiency $(P<0.05)$. In contrast, feed intake and water intake did not vary $(P>0.05)$ across the groups fed the different diets. Although body weight declined, there is no information on the composition of the body weight loss. These results were discussed fully elsewhere ${ }^{(10,20)}$.

\section{Analysis of mucosal cytokines}

$S$. enteritidis administration resulted in significantly $(P<0.05)$ higher pro-inflammatory IFN $\gamma, \mathrm{TNF} \alpha$ and lower anti-inflammatory IL-4, IL-10 mucosal concentrations for the challenged BD-fed rats compared with the unchallenged BD-fed rats in the jejunal, ileal, caecal and colonic mucosa. This effect was completely negated in the challenged rats receiving the FDOI-based diet. Challenged rats receiving the AOI diet were found to have similar $(P>0.05)$ pro- and anti-inflammatory cytokine concentrations when compared with the challenged rats fed the $\mathrm{BD}$ (Table 2).

\section{Analysis of mucosal immunoglobulins}

Mucosal rat IgA (total) concentrations were significantly $(P<0.05)$ greater in the challenged $\mathrm{BD}$ - and AOI-fed rats

Table 3. Intestinal mucosal $\operatorname{Ig} A$, IgG and IgE concentrations in unchallenged rats fed the basal diet $(\mathrm{BD})$ and rats challenged with Salmonella for $3 \mathrm{~d}$ and fed the $\mathrm{BD}$, freeze-dried ovine immunoglobulin (FDOI) diet or autoclaved ovine immunoglobulin (AOI) diet

(Mean values with their standard errors; $n$ 15)

\begin{tabular}{|c|c|c|c|c|c|c|}
\hline & \multirow{2}{*}{$\frac{\text { Unchallenged }}{\mathrm{BD}}$} & \multicolumn{3}{|c|}{ Challenged } & \multirow[b]{2}{*}{ SEM } & \multirow[b]{2}{*}{$P$} \\
\hline & & BD & FDOI & $\mathrm{AOI}$ & & \\
\hline \multicolumn{7}{|c|}{ Jejunal mucosa } \\
\hline $\lg A(g / l)$ & $0.74^{b}$ & $1.56^{\mathrm{a}}$ & $0.75^{b}$ & $1.50^{\mathrm{a}}$ & 0.021 & 0.026 \\
\hline $\lg G(g / l)$ & $0 \cdot 27$ & 0.28 & $0 \cdot 26$ & 0.28 & 0.024 & 0.248 \\
\hline $\lg E(\mathrm{mg} / \mathrm{l})$ & $95 \cdot 08$ & 93.58 & $90 \cdot 39$ & 95.55 & $19 \cdot 79$ & 0.286 \\
\hline \multicolumn{7}{|l|}{ lleal mucosa } \\
\hline $\lg A(g / l)$ & $0.69^{b}$ & $1 \cdot 61^{a}$ & $0.67^{b}$ & $1.57^{\mathrm{a}}$ & 0.022 & 0.031 \\
\hline $\lg G(g / l)$ & $281 \cdot 22$ & $297 \cdot 36$ & $259 \cdot 68$ & $287 \cdot 22$ & $26 \cdot 84$ & 0.311 \\
\hline $\lg E(m g / l)$ & 91.44 & $98 \cdot 94$ & $92 \cdot 75$ & $95 \cdot 91$ & $11 \cdot 29$ & 0.281 \\
\hline \multicolumn{7}{|c|}{ Caecal mucosa } \\
\hline $\lg A(g / l)$ & $84 \cdot 21^{b}$ & $192 \cdot 65^{\mathrm{a}}$ & $84 \cdot 38^{b}$ & $184 \cdot 15^{\mathrm{a}}$ & $29 \cdot 25$ & 0.006 \\
\hline $\lg G(g / l)$ & 58.49 & $64 \cdot 22$ & $54 \cdot 43$ & $69 \cdot 30$ & 11.58 & 0.290 \\
\hline $\lg E(m g / l)$ & $30 \cdot 44$ & 39.94 & $32 \cdot 75$ & 35.91 & $10 \cdot 29$ & 0.344 \\
\hline \multicolumn{7}{|c|}{ Colonic mucosa } \\
\hline $\lg A(g / l)$ & $89 \cdot 05^{b}$ & $206.53^{a}$ & $76 \cdot 36^{b}$ & $200 \cdot 56^{a}$ & $35 \cdot 4$ & 0.008 \\
\hline $\lg G(g / l)$ & $44 \cdot 38^{b}$ & $79 \cdot 95^{a}$ & $44 \cdot 99^{b}$ & $74 \cdot 58^{a}$ & $12 \cdot 35$ & 0.04 \\
\hline $\lg E(m g / l)$ & $30 \cdot 21$ & $36 \cdot 71$ & 31.52 & $33 \cdot 68$ & 9.58 & 0.274 \\
\hline
\end{tabular}


Table 4. Intestinal mucosal anti-Salmonella (ASE) IgA and ASE IgG concentrations (absorbance) in rats challenged with Salmonella for $3 \mathrm{~d}$ and fed the basal diet (BD), freeze-dried ovine immunoglobulin (FDOI) diet or autoclaved ovine immunoglobulin (AOI) diet

(Mean values with their standard errors; $n 15$ )

\begin{tabular}{lllllll}
\hline & \multicolumn{3}{c}{ Challenged } & & \\
\cline { 2 - 4 } & BD & FDOI & AOI & SEM & $P$ \\
\hline Jejunal mucosa & & & & & \\
ASE IgA (OD at 450 nm) & $0.46^{\mathrm{b}}$ & $0.96^{\mathrm{a}}$ & $0.48^{\mathrm{b}}$ & 0.09 & 0.006 \\
ASE IgG (OD at 450 nm) & 0.31 & 0.59 & 0.34 & 0.03 & 0.216 \\
Ileal mucosa & & & & & \\
ASE IgA (OD at 450 nm) & $0.44^{\mathrm{b}}$ & $0.91^{\mathrm{a}}$ & $0.49^{\mathrm{b}}$ & 0.08 & 0.003 \\
ASE IgG (OD at 450 nm) & 0.22 & 0.27 & 0.24 & 0.06 & 0.325 \\
Caecal mucosa & & & & & \\
ASE IgA (OD at 450 nm) & $0.48^{\mathrm{b}}$ & $0.85^{\mathrm{a}}$ & $0.49^{\mathrm{b}}$ & 0.06 & 0.004 \\
ASE IgG (OD at 450 nm) & $0.26^{\mathrm{b}}$ & $0.61^{\mathrm{a}}$ & $0.34^{\mathrm{b}}$ & 0.1 & 0.006 \\
Colonic mucOsa & & & & & \\
ASE IgA (OD at 450 nm) & $0.50^{\mathrm{b}}$ & $0.92^{\mathrm{a}}$ & $0.46^{\mathrm{b}}$ & 0.04 & 0.001 \\
ASE IgG (OD at 450 nm) & $0.48^{\mathrm{b}}$ & $0.85^{\mathrm{a}}$ & $0.44^{\mathrm{b}}$ & 0.08 & 0.006 \\
\hline
\end{tabular}

$\mathrm{OD}$, optical density.

a,b Mean values within a row with unlike superscript letters were significantly different $(P<0.05)$.

compared with the unchallenged BD-fed rats in all the segments (jejunal, ileal, caecal and colonic). This effect was not evident in the challenged rats fed the FDOI diet. Consumption of the different diets (BD, FDOI and AOI) did not affect mucosal IgG (in jejunum, ileum and caecum, $P=0 \cdot 25-0 \cdot 31$ ) concentrations. However, in the colon, rats challenged and fed either the BD or AOI diet had significantly $(P<0.05)$ higher mucosal IgG than for the unchallenged rats receiving the $\mathrm{BD}$. There was no significant $(P>0.05)$ difference between the unchallenged BD-fed rats and the challenged FDOI-fed rats. Mucosal IgE levels were not affected $(P=0 \cdot 27-0 \cdot 34)$ by treatment (Table 3$)$.

\section{Quantitative analysis of mucosal anti-Salmonella immunog/obulins}

In all intestinal mucosal segments (jejunal, ileal, caecal and colonic), ASE IgA levels were significantly $(P<0.05)$ enhanced for rats (challenged with $S$. enteritidis) fed the FDOI diet compared with the other groups. A similar trend was evident for the levels of ASE IgG in the mucosal segments (except in jejunum and ileum) where the challenged rats fed the FDOI diet had significantly $(P<0.05)$ higher levels of ASE IgG compared with the other groups (Table 4).

\section{Determination of myeloperoxidase activity}

Challenging rats with $S$. enteritidis resulted in significantly $(P<0.05)$ higher MPO activity in all of the intestinal mucosal segments for the rats receiving the $\mathrm{BD}$ compared with the unchallenged BD-fed rats. There was no significant $(P>0.05)$ difference between the challenged AOI-fed rats and the challenged BD-fed rats. This effect was not seen in the challenged rats fed the FDOI diet. MPO activity in the challenged rats receiving the FDOI diet did not differ from those from the unchallenged rats (Table 5).
Mean organ, mesenteric lymph node weights and number of ileal Peyer's patches

Mean pancreas weight relative to empty body weight and MLN weights and the number of ileal Peyer's patches for the rats on the final day (day 18) of the study are shown in Table 6. Challenging with $S$. enteritidis resulted in significantly $(P<0.05)$ higher MLN and pancreas weights for the rats receiving the BD. Moreover, pancreas and MLN weights in the challenged rats receiving the AOI diet were not significantly $(P>0.05)$ different from the challenged rats fed the BD. In contrast, the mean pancreas and MLN weights in the rats challenged but receiving the FDOI diet were not significantly $(P>0.05)$ different from the unchallenged rats receiving the $\mathrm{BD}$, but were significantly $(P<0.05)$ different from the challenged $\mathrm{BD}$ - and AOI diet-fed rats. A similar trend was observed for the number of ileal Peyer's patches.

\section{Quantitative analysis of mucin}

Data for the amount of mucin in the intestinal mucosal scrapings are shown in Table 7. In the jejunum, ileum, caecum and colon, intestinal mucosal mucin protein content was significantly $(P<0.05)$ higher for the unchallenged rats fed the $\mathrm{BD}$ than for the rats challenged and receiving either the $\mathrm{BD}$ or AOI diet. However, in all of the segments, mucosal mucin protein content was significantly $(P<0.05)$ higher for the challenged rats fed the FDOI diet than for the challenged rats fed either the BD or AOI diet, but was not different $(P>0.05)$ from the unchallenged rats fed the BD.

\section{Discussion}

In mammals, binding of $S$. enteritidis to the intestinal mucosa during intestinal infection is known to elicit the production of pro-inflammatory cytokines such as IFN $\gamma$, TNF- $\alpha$ (T-helper (Th) 1 cytokines), IL1 and IL-6, chemokines such as IL-8, but to down-regulate Th2 cell cytokines such as IL-4 and IL-10 ${ }^{(21,22)}$. IFN $\gamma$ and TNF $\alpha$ have been shown to suppress the invasiveness of Salmonella into the host cells ${ }^{(23)}$. Generally, Th1 cytokines primarily facilitate cellular immunity

Table 5. Intestinal mucosal myeloperoxidase (MPO) activity (U/I $\left.{ }^{\star}\right)$ for the unchallenged rats fed the basal diet (BD) and rats challenged with Salmonella for $3 \mathrm{~d}$ and fed the BD, freeze-dried ovine immunoglobulin (FDOI) diet or autoclaved ovine immunoglobulin (AOI) diet

(Mean values with their standard errors; $n$ 15)

\begin{tabular}{|c|c|c|c|c|c|c|}
\hline & \multirow{2}{*}{$\frac{\text { Unchallenged }}{\mathrm{BD}}$} & \multicolumn{3}{|c|}{ Challenged } & \multirow[b]{2}{*}{ SEM } & \multirow[b]{2}{*}{$P$} \\
\hline & & BD & FDOI & $\mathrm{AOI}$ & & \\
\hline Jejunum & $5.57^{b}$ & $15 \cdot 78^{a}$ & $6 \cdot 08^{b}$ & $15 \cdot 82^{a}$ & 1.03 & 0.022 \\
\hline Ileum & $6 \cdot 74^{b}$ & $15 \cdot 75^{a}$ & $6.99^{b}$ & $14.98^{a}$ & 1.32 & 0.021 \\
\hline Caecum & $6.99^{b}$ & $18 \cdot 84^{a}$ & $7 \cdot 14^{b}$ & $18 \cdot 36^{a}$ & 1.26 & 0.019 \\
\hline Colon & $7 \cdot 82^{b}$ & $18 \cdot 41^{a}$ & $7 \cdot 85^{b}$ & $14.65^{a}$ & 1.01 & 0.015 \\
\hline
\end{tabular}

a,b Mean values within a row with unlike superscript letters were significantly different $(P<0.05)$

* One unit of MPO is defined as the amount of MPO that hydrolyses the substrate and generates taurine chloramine to consume $1.0 \mu \mathrm{mol}$ trinitrobenzene (TNB) per min at $25^{\circ} \mathrm{C}$. 
Table 6. Mean mesenteric lymph node (MLN) weights and relative pancreas weights and number of ileal Peyer's patches $(n 8)$ for the unchallenged rats fed the basal diet (BD) and rats challenged with Salmonella for $3 \mathrm{~d}$ and fed the BD, freeze-dried ovine immunoglobulin (FDOI) diet or autoclaved ovine immunoglobulin (AOI) diet

(Mean values with their standard errors; $n 15$ )

\begin{tabular}{|c|c|c|c|c|c|c|}
\hline & \multirow{2}{*}{$\frac{\text { Unchallenged }}{\mathrm{BD}}$} & \multicolumn{3}{|c|}{ Challenged } & \multirow[b]{2}{*}{ SEM } & \multirow[b]{2}{*}{$P$} \\
\hline & & $\mathrm{BD}$ & FDOI & $\mathrm{AOI}$ & & \\
\hline Ileal Peyer's patches* & $5 \cdot 14^{\mathrm{b}}$ & $13 \cdot 29^{a}$ & $5 \cdot 23^{\mathrm{b}}$ & $12 \cdot 05^{\mathrm{a}}$ & 0.097 & 0.012 \\
\hline MLN (g) & $0.89^{\mathrm{b}}$ & $1.45^{\mathrm{a}}$ & $0.90^{\mathrm{b}}$ & $1.44^{\mathrm{a}}$ & 0.028 & 0.024 \\
\hline Pancreas ( $\mathrm{g} / 100 \mathrm{~g}$ body weight) & $0 \cdot 28^{b}$ & $0.45^{\mathrm{a}}$ & $0.29^{b}$ & $0.43^{\mathrm{a}}$ & 0.005 & 0.011 \\
\hline
\end{tabular}

${ }^{a, b}$ Mean values within a row with unlike superscript letters were significantly different $(P<0.05)$ ${ }^{*} n 8$.

against intracellular bacteria and viruses, thereby activating cellular mechanisms that end in phagocyte-dependent inflammation $^{(24)}$. On the other hand, Th2 cytokines prevent several functions of phagocytic cells, regulate humoral immunity, regulate or suppress inflammation, and promote phagocyte-independent inflammation ${ }^{(24)}$. The primary antibacterial effect of IFN $\gamma$ seems to be the suppression of bacterial growth by induction of $\mathrm{NO}^{(25)}$. In the present study, mucosal IFN $\gamma$ and TNF $\alpha$ concentrations were significantly lower for the challenged rats fed the FDOI diet when compared with the challenged rats fed the $\mathrm{BD}$, suggesting that the colonisation of foodborne pathogens might be inhibited through enhanced luminal growth suppression of $S$. enteritidis in the challenged rats fed the FDOI compared with the challenged rats fed the BD or AOI diet. These findings confirm some of our earlier results ${ }^{(10)}$ and those of others ${ }^{(26)}$ where plasma IFN $\gamma$, TNF $\alpha$, IL-10 and IgA concentrations were significantly lower for challenged rats fed an FDOI diet when compared with challenged rats fed the $\mathrm{BD}$, suggesting that there was a higher translocation of $S$. enteritidis into the blood of the challenged rats fed the BD than for the challenged rats fed the FDOI diet. Intestinal mucosal IL- 4 and IL-10 concentrations were significantly higher for the challenged FDOI-fed rats compared with the other challenged diet groups. This is contradictory to our earlier findings where we showed lower plasma IL-10 levels for the challenged FDOI-fed rats ${ }^{(14)}$, but in the presently reported study the higher intestinal IL-10 levels may be due to a high enrichment of Lactobacillus strains in the intestinal segments (observed in previous studies) which have been shown to up-regulate intestinal tissue IL-10 ${ }^{(27)}$. FDOI prevented the deleterious effects of $S$. enteritidis on the release of mucosal pro-inflammatory cytokines, suggesting that the effects of immunoglobulin supplements during an inflammatory event involve changes in the mucosal cytokine balance.

Intestinal rat IgA protects mucous membranes by preventing bacteria from attaching to the mucosal surface and works in a process known as immune exclusion, where intestinal IgA binds to the bacteria, pathogen or its antigen, thereby preventing its translocation ${ }^{(28)}$. In the present study, the feeding of FDOI led to significantly $(P<0.05)$ lower mucosal IgA concentrations in all of the intestinal segments compared with the challenged rats fed either the $\mathrm{BD}$ or AOI diet. This suggests that the pathogen burden was greatly reduced in the rats fed the FDOI. A similar result (but only in colonic mucosa) was observed with rat mucosal IgG concentrations. Conversely, we found higher mucosal ASE IgA and ASE IgG in the challenged FDOI-fed rats, showing that feeding the FDOI diet up-regulated specific humoral immune responses against $S$. enteritidis, perhaps by increasing either $\mathrm{B}$ cell proliferation or differentiation of $\mathrm{B}$ cells to secrete ASE IgA and ASE IgG antibodies ${ }^{(10,14,29)}$. Rodenburg et al. ${ }^{(30)}$ using a microarray technique reported that immunity genes in ileal and colonic mucosal segments were up-regulated from day 1 of $S$. enteritidis challenge, which we have also observed $^{(14)}$.

The evidence of infection-induced body weight loss in the challenged rats fed the $\mathrm{BD}$ or $\mathrm{AOI}$ diet also confirms that the systemic infection was more severe in those groups compared with the challenged rats receiving the FDOI diet ${ }^{(20)}$. Neutrophils are key cellular components in the inflammatory response and play a key role in host defence against bacterial infections and disease through oxygen-dependent and -independent bactericidal mechanisms ${ }^{(31)}$. A high leucocyte (WBC) count along with high neutrophil count can also indicate a systemic translocation of $S$. enteritidis. In our previous study, neutrophils and leucocyte counts were lower $(P<0.05)$ in $S$. enteritidis-challenged rats fed the FDOI diet when compared with challenged rats fed either the $\mathrm{BD}$ or AOI diet. Consistent with the stimulated translocation of $S$. enteritidis in the challenged rats fed either the $\mathrm{BD}$ or $\mathrm{AOI}$ diet in the present study, the mucosal inflammatory response (MPO activity) in the jejunal, ileal, caecal and colonic mucosa

Table 7. Intestinal mucosal mucin protein concentrations for the unchallenged rats fed the basal diet (BD) and rats challenged with Salmonella for $3 \mathrm{~d}$ and fed the $\mathrm{BD}$, freeze-dried ovine immunoglobulin (FDOI) diet or autoclaved ovine immunoglobulin (AOI) diet

(Mean values with their standard errors; $n$ 15)

\begin{tabular}{|c|c|c|c|c|c|c|}
\hline & \multirow{2}{*}{$\frac{\text { Unchallenged }}{\text { BD }}$} & \multicolumn{3}{|c|}{ Challenged } & \multirow[b]{2}{*}{ SEM } & \multirow[b]{2}{*}{$P$} \\
\hline & & $\mathrm{BD}$ & FDOI & $\mathrm{AOI}$ & & \\
\hline Jejunum ( $\mu \mathrm{g} / \mathrm{l})$ & $32 \cdot 17^{a}$ & $12 \cdot 78^{b}$ & $38.68^{a}$ & $13 \cdot 82^{b}$ & 9.63 & 0.03 \\
\hline lleum (mg/l) & $3 \cdot 72^{\mathrm{a}}$ & $0.75^{\mathrm{b}}$ & $4.01^{a}$ & $0.98^{b}$ & 1.01 & 0.04 \\
\hline Caecum $(\mu \mathrm{g} / \mathrm{l})$ & $13 \cdot 25^{a}$ & $6 \cdot 84^{\mathrm{b}}$ & $13 \cdot 14^{a}$ & $6 \cdot 36^{b}$ & 1.56 & 0.03 \\
\hline Colon $(\mu \mathrm{g} / \mathrm{l})$ & $12 \cdot 17^{\mathrm{a}}$ & $5 \cdot 41^{\mathrm{b}}$ & $13 \cdot 65^{a}$ & $5.65^{b}$ & $2 \cdot 01$ & 0.02 \\
\hline
\end{tabular}

${ }^{\mathrm{a}, \mathrm{b}}$ Mean values within a row with unlike superscript letters were significantly different $(P<0.05)$. 
was significantly $(P<0.05$; about two-fold increase) higher compared with challenged rats fed the FDOI diet (Table 5). MPO is a marker of neutrophil accumulation ${ }^{(32)}$ and inflammation ${ }^{(33)}$. The observed inhibition of enhanced intestinal mucosal MPO activity by ovine serum immunoglobulins (FDOI) in the $S$. enteritidis-challenged rats clearly suggests a potent intestinal anti-inflammatory effect against intestinal injury. Previously, we reported that mucosal inflammation (in jejunal, ileal and colonic segments) was not evident in $S$. enteritidis-challenged rats fed the FDOI diet, based on measures of gut morphology and histology. However, mucosal inflammation in all of the intestinal segments was notably severe in the BD and AOI groups after Salmonella challenge $^{(20)}$.

Entry of the entero-invasive salmonellae into the gut tissues is known to occur by colonising of the intestinal epithelium and Peyer's patches (via M cells), passing through the submucosa and arriving at the MLN via the draining lymphatic vessels. From this point, the blood and other vital organs such as the liver and spleen may be colonised. Any damage to the integrity of the epithelium may initiate an opportunity for early access by the organisms ${ }^{(34)}$. In the present study, the number of Peyer's patches was significantly $(P<0.05)$ lower in the challenged rats fed the FDOI diet when compared with the challenged rats fed either the BD or AOI diet (Table 6). In animal models, a reduced number of Peyer's patches has been linked to a higher resistance to orally acquired infection ${ }^{(35)}$. Reports have also shown that pathogenic bacteria like Streptococcus or Salmonella may increase the number of $M$ cells within the Peyer's patches which might increase the bacterial translocation ${ }^{(36)}$. Moreover, weights of the MLN and pancreas increased significantly $(P<0.05)$ in the challenged rats fed either the BD or AOI diet compared with the challenged rats fed the FDOI diet or unchallenged rats fed the BD (Table 6). Some of the present results are similar to the findings of Renner et al. ${ }^{(37)}$ where they reported a high incidence of acute pancreatitis in adult patients with Salmonella infection ${ }^{(38)}$. This is also supported by findings of severe acute pancreatitis during Salmonellosis, suggesting that serious pancreatic disease may represent a complication of Salmonella infection ${ }^{(39)}$.

Optimal functioning of the mucosal barrier is vital to prevent bacterial translocation and gut-derived septicaemia ${ }^{(40)}$. The decreased intestinal mucosal mucin content observed in the challenged rats fed either the $\mathrm{BD}$ or $\mathrm{AOI}$ diet compared with the challenged rats receiving the FDOI diet (Table 7) also suggests damage to the gut mucosa or at least potentially compromised mucosal protection. These results are similar to our recent findings ${ }^{(10,20)}$ where we reported that rats challenged with $S$. enteritidis and fed the FDOI diet had significantly $(P<0.05)$ higher secreted mucin in both ileal and colonic luminal segments. King et al. ${ }^{(41)}$ also reported that feeding pigs with dried plasma resulted in an increase of gut mucin. Mucin acts as the main structural component of the mucus layer, giving rise to its polymeric, viscoelastic and protective properties. The mesh-like structure of the mucin gel prevents the entry of enteric pathogens and also serves numerous other functions, such as lubrication for the passage of particulate matter; preservation of a hydrated layer over the epithelium; providing a barrier to noxious substances; and forming a permeable gel layer, for the exchange of gases and nutrients, with the underlying epithelium ${ }^{(42)}$. Caballero-Franco et al. ${ }^{(43)}$ reported that the Lactobacillus species (VSL\#3 probiotic formula) were the strongest potentiator of mucin secretion in vitro. In the present study, mucin protein was found to be higher in the jejunal, ileal, caecal and colonic mucosa of the challenged rats fed the FDOI. In our earlier studies, we observed that feeding rats an FDOI-containing diet resulted in an increase in the total Lactobacillus, Lactobacillus johnsonii and L. acidophillus and depletion of enterobacteria in both ileal and colonic luminal segments ${ }^{(10,14)}$. Thus, feeding the FDOI diet may have resulted in an increase in the number of lactic acid bacteria which may have potentiated the mucosal mucin protein in vivo.

An FDOI diet supported enhanced mucosal immunomodulation when compared with a control diet or with a diet containing AOI (negative control). Given that the immunoglobulin in the AOI diet was inactivated as confirmed by circular dichroism spectral analysis (data not shown), it would suggest that the active immunoglobulin present in the FDOI was responsible for the greater mucosal immunity. An understanding of the detailed mechanistic basis of the effects of FDOI on mucosal immunity awaits future research. However, the results reported in the present study suggest a mechanism by which intact ovine serum immunoglobulins (and not the autoclaved ovine immunoglobulins) may influence the positive effect. Passive inhibition of the adhesion of the pathogenic bacteria to the host intestinal wall may be mediated by antibodies present in FDOI $^{(44)}$. Lambs are exposed to a much harsher environment than the average human. As a result, it is possible that the animal's immune system is better adjusted to counterattacking the full spectrum of pathogens by producing antibodies against them. It is well known that animal serum contains antibodies that are effective against a variety of pathogens like Escherichia coli, Salmonella, Listeria, C. difficile, rotavirus, and many more ${ }^{(2,7,10,44)}$. Therefore, intact ovine serum immunoglobulins (FDOI) may provide passive antimicrobial protection by exclusion of opportunistic pathogens, thereby resulting in stronger mucosal immunity. This was not seen with the AOI, as the antibodies present in the AOI fraction were denatured due to heat inactivation.

In conclusion, FDOI selectively modulated the mucosal immune function of rats through enhanced specific immune responses, such as by increasing the production of antiinflammatory cytokines, anti-Salmonella immunoglobulins and mucin. It also decreased the production of pro-inflammatory cytokines, lowering the MPO activity and non-specific IgA and $\mathrm{IgG}$ in the intestinal mucosa. Overall, orally administered FDOI significantly reduced the negative effects of $S$. enteritidis challenge in the growing rat potentially by passive prevention of attachment and translocation of $S$. enteritidis. These effects resulted in the rat having an increased resistance to infection. Autoclaving the ovine immunoglobulin fraction leading to denaturation of the immunoglobulins, nullified the immunoglobulin effect. 


\section{Acknowledgements}

The authors' responsibilities were as follows: P. B. and P. J. M. designed the study; P. B. conducted the research; P. J. M. supervised the research; P. B. analysed and interpreted the results; P. B. wrote the paper; P. B. had primary responsibility for the final content. Both authors read and approved the final manuscript.

P. B. was the recipient of a Riddet Institute doctoral scholarship for his $\mathrm{PhD}$ research studies. The authors are grateful to the Riddet Institute, Palmerston North, New Zealand, for providing experimental facilities and financial support for this research project.

The authors declare no conflicts of interest.

\section{References}

1. Späth JP (1999) Structure and function of immunoglobulins Sepsis 3, 197-218.

2. Pierce JL, Cromwell GL, Lindemann MD, et al. (2005) Effects of spray-dried animal plasma and immunoglobulins on performance of early weaned pigs. J Anim Sci 83, 2876-2885.

3. Campbell JM, Polo J, Russell LE, et al. (2010) Review of spray-dried plasma's impact on intestinal barrier function. Livest Sci 133, 239-241.

4. Coffey RD \& Cromwell GL (1995) The impact of the environment and antimicrobial agents on the growth response of early-weaned pigs to spray-dried porcine plasma. J Anim Sci 73, 2532-2539.

5. Lallès JP, Bosi P, Janczyk P, et al. (2009) Impact of bioactive substances on the gastrointestinal tract and performance of weaned piglets: a review. Animal 3, 1625-1643.

6. Fiocchi C (1997) Intestinal inflammation: a complex interplay of immune and nonimmune cell interactions. Am J Physiol 273, G769-G775.

7. Eibl MM, Wolf HM, Furnkranz H, et al. (1988) Prevention of necrotizing enterocolitis in low-birth-weight infants by IgAIgG feeding. $N$ Engl J Med 319, 1-7.

8. Hammarström V, Smith CIE \& Hammarström L (1993) Oral immunoglobulin treatment in Campylobacter jejuni enteritis. Lancet 341, 1036.

9. Tjellström B, Stenhammar L, Eriksson S, et al. (1993) Oral immunoglobulin A supplement in treatment of Clostridium difficile enteritis. Lancet 341, 701-702.

10. Balan P (2011) Effects of orally administered ovine serum immunoglobulin in the normal and Salmonella enteritidischallenged growing rat. PhD Thesis, Massey University.

11. Balan P, Han KS, Rutherfurd SM, et al. (2009) Orally administered ovine serum immunoglobulins influence growth performance, organ weights, and gut morphology in growing rats. J Nutr 139, 244-249.

12. Balan P, Han KS, Rutherfurd-Markwick K, et al. (2010) Immunomodulatory effects of ovine serum immunoglobulin in the growing rat. Animal 4, 1702-1708.

13. Balan P, Han KS, Singh H, et al. (2011) Dietary supplementation with ovine serum immunoglobulin is associated with increased gut mucin secretion in the growing rat. Animal 5, 1916-1922.

14. Balan P, Han KS, Rutherfurd-Markwick K, et al. (2011) Immunomodulatory effects of ovine serum immunoglobulin in growing rats gavaged with Salmonella enteritidis. J Nutr 141, 950-956.

15. Pérez-Bosque A, Miró L, Polo J, et al. (2010) Dietary plasma protein supplements prevent the release of mucosal proinflammatory mediators in intestinal inflammation in rats. J Nutr 140, 25-30.

16. Vermeer AW \& Norde W (2000) The thermal stability of immunoglobulin: unfolding and aggregation of a multidomain protein. Biophys J 78, 394-404.

17. National Research Council (1995) Nutrient Requirements of Laboratory Animals, 4th ed. Washington, DC: National Academy Press.

18. AOAC International (2003) Official Methods of Analysis of $A O A C$ International, 17th ed., 2nd revision. Gaithersburg, MD: Association of Analytical Communities.

19. Deng SX, Cheng AC, Wang MS, et al. (2007) Gastrointestinal tract distribution of Salmonella enteritidis in orally infected mice with a species-specific fluorescent quantitative polymerase chain reaction. World J Gastroenterol 13, 6568-6574.

20. Balan P, Han KS, Rutherfurd SM, et al. (2011) Dietary supplementation with ovine serum immunoglobulin attenuates acute effects on growth, organ weights, gut morphology and intestinal mucin production in the growing rat challenged with Salmonella enteritidis. Animal 5, 1570-1578.

21. Eckmann L \& Kagnoff MF (2001) Cytokines in host defence against Salmonella. Microbes Infect 3, 1191-1200.

22. Coburn B, Grassl GA \& Finlay BB (2007) Salmonella, the host and disease: a brief review. Immunol Cell Biol 85, 112-118.

23. Degré M, Bukholm G \& Czarniecki CW (1989) In vitro treatment of HEp-2 cells with human tumor necrosis factor- $\alpha$ and human interferons reduces invasiveness of Salmonella typhimurium. J Biol Regul Homeost Agents 3, 1-7.

24. Corthay A (2006) A three-cell model for activation of naive T helper cells. Scand J Immunol 64, 93-96.

25. Makela PH \& Hormaeche CE (1996) Immunity to Salmonella. In Host Response to Intracellular Pathogens, pp. 143-161 [SHE Kaufmann, editor]. New York: Chapman \& Hall.

26. Stoycheva M \& Murdjeva M (2005) Serum levels of interferon- $\gamma$, interleukin-12, tumour necrosis factor- $\alpha$, and interleukin-10, and bacterial clearance in patients with gastroenteric Salmonella infection. Scand J Infect Dis 37, $11-14$.

27. Perdigón G, Maldonado Galdeano C, Valdez JC, et al. (2002) Interaction of lactic acid bacteria with the gut immune system. Eur J Clin Nutr 56, Suppl. 4, S21-S26.

28. Amin PB, Diebel LN, Liberati DM, et al. (2007) T-cell cytokines affect mucosal immunoglobulin A transport. Am J Surg 194, 128-133.

29. Jain S, Yadav H \& Sinha PR (2009) Probiotic dahi containing Lactobacillus casei protects against Salmonella enteritidis infection and modulates immune response in mice. $J$ Med Food 12, 576-583.

30. Rodenburg W, Keijer J, Kramer E, et al. (2007) Salmonella induces prominent gene expression in the rat colon. $B M C$ Microbiol 12, 84-100.

31. Elsbach P \& Weiss J (1992) Oxygen-independent antimicrobial systems of phagocytes. In Inflammation: Basic Principles and Clinical Correlates, pp. 603-636 [JI Gallin, IM Goldstein and R Snyderman, editors]. New York: Raven Press

32. Sener G, Sehirli O, Cetinel S, et al. (2005) Amelioration of sepsis-induced hepatic and ileal injury in rats by the leukotriene receptor blocker montelukast. Prostaglandins Leukot Essent Fatty Acids 73, 453-462.

33. Nieto N, Fernandez MI, Torres MI, et al. (1988) Dietary monounsaturated $n-3$ and $n-6$ long-chain polyunsaturated fatty acids affect cellular antioxidant defence system in rats with experimental ulcerative colitis induced by trinitrobenzene sulfonic acid. Dig Dis Sci 43, 2676-2687. 
34. Islam AF, Moss ND, Dai Y, et al. (2000) Lipopolysaccharideinduced biliary factors enhance invasion of Salmonella enteritidis in a rat model. Infect Immun 68, 1-5.

35. Prinz M, Huber G, Macpherson AJ, et al. (2003) Oral prion infection requires normal numbers of Peyer's patches but not of enteric lymphocytes. Am J Path 162, 1103-1111.

36. Meynell HM, Thomas NW, James PS, et al. (1998) Up-regulation of microsphere transport across the follicle-associated epithelium of Peyer's patch by exposure to Streptococcus pneumoniae R36a. FASEB J 13, 611-619.

37. Renner F, Nimeth C \& Demmelabauer R (1991) High frequency of concomitant pancreatitis in Salmonella enteritidis. Lancet 337, 1611.

38. Tositti G, Fabris P, Barnes E, et al. (2001) Pancreatic hyperamylasemia during acute gastroenteritis: incidence and clinical relevance. BMC Infect Dis 1, 18.

39. Hamaguchi H, Okabayashi Y, Yoneda R, et al. (1999) A case of acute pancreatitis complicating Salmonella enteritis. Int J Pancreatol 26, 189-192.
40. Moncada DM \& Chadee K (2002) Production, structure, and function of gastrointestinal mucins. In Infections of the Gastrointestinal Tract, pp. 57-79 [MJ Blaser, editor]. Philadelphia: Lippincott.

41. King MR, Morel PCH, Pluske JR, et al. (2008) A comparison of the effects of dietary spray-dried bovine colostrum and animal plasma on growth and intestinal histology in weaner pigs. Livest Sci 119, 167-173.

42. Laboisse C, Jarry A, Branka JE, et al. (1996) Recent aspects of the regulation of intestinal mucus secretion. Proc Nutr Soc 55, 259-264.

43. Caballero-Franco C, Keller K, De Simone C, et al. (2007) The VSL \#3 probiotic formula induces mucin gene expression and secretion in colonic epithelial cells. Am J Physiol Gastrointest Liver Physiol 292, G315-G322.

44. Han KS, Boland M, Singh H, et al. (2009) The in vitro antipathogenic activity of immunoglobulin concentrates extracted from ovine blood. Appl Biochem Biotechnol 157, $442-452$. 